\title{
Prevalencia de la infección por Helicobacter pylori en población sana en la Comunidad de Madrid
}

\author{
F. Sánchez Ceballos, C. Taxonera Samsó, M. García Alonso, C. Alba López, L. Sainz de los Terreros \\ Soler ${ }^{1}$ y M. Díaz-Rubio
}

Servicio de Aparato Digestivo. Hospital Clínico San Carlos. ${ }^{I}$ Servicio de Medicina Preventiva. Hospital Ramón y Cajal. Madrid

\section{RESUMEN}

Objetivo: conocer la prevalencia de la infección por Helicobacter pylori en población sana en la Comunidad de Madrid.

Material y métodos: estudio transversal descriptivo en el que se realiza el diagnóstico de la infección por Helicobacter pylori mediante la prueba del test del aliento con ${ }^{13} \mathrm{C}$-urea.

Resultados: se estudian un total de 618 sujetos. De estos, 481 son considerados evaluables con una prevalencia de infección por Helicobacter pylori del 60,3\%. En esta cohorte el $36,4 \%$ eran varones y el $63,6 \%$ mujeres con una prevalencia del 60,6 y $60,1 \%$ respectivamente sin diferencias significativas. La mediana de edad de los pacientes evaluados fue de 37,5 años (rango 4-82) estableciéndose que existe una relación lineal con significación estadística entre la infección por Helicobacter pylori y la edad $\left(\chi^{2}\right.$ lineal $\left.=33,31 ; p<0,001\right)$ : a mayor edad mayor probabilidad de infección. La prevalencia aumenta con la edad siendo máxima entre los 60 y 69 años (83,3\% infectados). En 169 sujetos $(35,1 \%)$ se conoce el nivel de estudios sin que exista relación entre este y la infección por Helicobacter pylori.

Conclusión: el estudio muestra que la prevalencia de la infección por Helicobacter pylori en población sana en Madrid es elevada $(60,3 \%)$, y aumenta progresivamente a lo largo de la vida para alcanzar un pico máximo en la $6^{\underline{a}}$ década. No se encuentran diferencias en la muestra en función del sexo ni del nivel de estudios.

Palabras clave: Helicobacter pylori. Prevalencia. Epidemiología. Test del aliento.

\begin{abstract}
Objective: to determine the prevalence of Helicobacter pylori infection in the healthy population of Madrid Autonomous Community (AC).

Material and methods: a descriptive, cross-sectional study where Helicobacter pylori infection is diagnosed by means of the ${ }^{13} \mathrm{C}$-urea breath test.

Results: a total of 618 subjects were studied. Among these, 481 were considered evaluable with a prevalence of Helicobacter pylori infection of $60.3 \%$. In this cohort $36.4 \%$ were men and $63.6 \%$ were women, with a prevalence of 60.6 and $60.1 \%$, respectively, and no relevant differences between both subgroups. The median age of patients was 37.5 years (range 4-82), and a statistically significant linear relationship between Helicobacter pylori infection and age (linear $\chi^{2}=33.31 ; p<0.001$ ) was established-chances of infection increase with age. Prevalence increases with age and peaks at 60 to 69 years (83.3\% infected). For 169 subjects (35.1\%) education level was unknown, and no relationship between this level and Helicobacter pylori infection was found.

Conclusions: the study shows that the prevalence of Helicobacter pylori infection in the healthy population of Madrid AC is high (60.3\%), progressively increases with age, and peaks in people in their sixties. The sample showed no differences regarding sex or education level.
\end{abstract}

Key words: Helicobacter pylori. Prevalence. Epidemiology. Breath test.

Sánchez Ceballos F, Taxonera Samsó C, García Alonso M, Alba López C, Sainz de los Terreros Soler L, Díaz-Rubio M. Prevalencia de la infección por Helicobacter pylori en población sana en la Comunidad de Madrid. Rev Esp Enferm Dig 2007; 99: 497-501.

\section{INTRODUCCIÓN}

En la década de los 80 las aportaciones de Warren y Marshall en relación con Helicobacter pylori $(\mathrm{Hp})(1)$

Recibido: 21-05-07.

Aceptado: 30-05-07.

Correspondencia: Francisco Sánchez Ceballos. C/ Velacho Bajo, 8, 6A. 28033 Madrid.e-mail: fsceballos@hotmail.com hacen que en años posteriores se modifiquen los conceptos fisiopatológicos y terapéuticos de distintos procesos gastroduodenales. Este microorganismo está directamente involucrado en la gastritis crónica, adenocarcinoma o linfoma MALT gástricos y revolucionó la patogenia e historia natural de la úlcera gastroduodenal. Sin embargo la mayoría de los individuos infectados nunca tendrán manifestaciones clínicas sin que podamos actualmente identificar a la población de riesgo de desarrollar patología asociada al $H p(2,3)$. 
Al igual que ocurre con otras enfermedades con alta penetrancia es difícil establecer las cifras de prevalencia exactas, pero se estima que aproximadamente el $50 \%$ de la población mundial está infectada por este microorganismo (4). Se han realizado multitud de estudios de prevalencia con resultados dispares y con una frecuente limitación metodológica, dado que los pacientes generalmente pertenecen al entorno sanitario con el consiguiente sesgo de selección. Por la gran variedad de las cifras de prevalencia y porque el importar resultados de otras áreas puede llevar a magnificar o minimizar las medidas a tomar, es importante conocer las tasas de la infección en nuestro medio. El objetivo del presente trabajo es determinar la prevalencia de la infección por $\mathrm{Hp}$ en población sana en Madrid.

\section{MATERIAL Y MÉTODO}

\section{Sujetos}

En la Feria Internacional de la Salud (FISALUD) celebrada en Madrid en 2004, 2005 y 2006, la Sociedad Española de Patología Digestiva (SEPD) participó con un stand informativo sobre distintos aspectos relacionados con el aparato digestivo y sus afecciones. Además se ofreció la oportunidad de realizar voluntariamente la determinación de $H p$ a los asistentes al evento. Se entrevista a los participantes recogiendo: edad, sexo, antecedente de úlcera gástrica o duodenal, consumo de antibiótico o inhibidores de la bomba de protones (IBP) en el último mes, tratamiento erradicador previo para $\mathrm{Hp}$, nivel de estudios y lugar de residencia.

Consideramos población sana, y por lo tanto evaluable, aquella sin antecedente de úlcera, duodenitis erosiva, cirugía gastroduodenal o tratamiento erradicador previo. Se han considerado los siguientes criterios de exclusión: a) antecedentes de úlcera duodenal o gástrica, duodenitis erosiva o cirugía gastroduodenal; b) consumo de antibióticos o IBP en el mes previo; c) tratamiento erradicador previo; d) incapacidad psíquica para comprender la naturaleza de la prueba; y e) negativa a la participación en el estudio.

\section{Diagnóstico de la infección por Helicobacter pylori}

El diagnóstico de la infección por $H p$ se realizó mediante el test del aliento con urea marcada con carbono 13. Se procede a la toma de una muestra de aliento basal y otra tras la administración de $100 \mathrm{mg}$ de urea-C $\mathrm{C}^{13}$ (UBTest $^{\circledR}$, Otsuka Pharmaceutical, España). Las muestras se analizan mediante espectrofotometría de infrarrojos (UBiTIR300, Otsuka Pharmaceutical, España). El aumento del ${ }^{13} \mathrm{C}$ en el aliento se expresa como una diferencia absoluta entre el cociente ${ }^{13} \mathrm{C} /{ }^{12} \mathrm{C}$ antes de la administración (toma basal) y 20 minutos después de la toma de urea- ${ }^{13} \mathrm{C}$. Si el valor $\Delta^{13} \mathrm{CO}_{2}$ es igual o superior a $2,5 \%$ el test es positivo y el sujeto se considera infectado por $\mathrm{Hp}$. El método muestra una sensibilidad del 98,2\% (IC95\%: 94,8-99,6\%) y una especificidad del 97,9\% (IC95\%: 88,9-99,9\%) cuando se compara con un patrón de referencia basado en métodos invasivos, según consta en ficha técnica.

\section{Análisis estadístico}

Se trata de un estudio transversal descriptivo. Los datos se introdujeron en una base de datos utilizando el programa Access $^{\circledast}$ (para Windows) y el análisis estadístico se llevó a cabo con el programa SPSS v.12.0. La medida de frecuencia utilizada ha sido la prevalencia.

Se comprobó mediante la prueba de KolmogorovSmirnov y mediante análisis gráfico que la edad de la población estudiada no seguía una distribución normal, por lo que para su análisis se utilizaron pruebas no paramétricas (U de Mann-Whitney). Para la comparación de variables discretas se utilizó la $\chi^{2}$ y el contraste chi-cuadrado de Mantel-Haenszel. El nivel de significación estadística se estableció en $\mathrm{p}<0,05$.

\section{RESULTADOS}

Se realizaron un total de 618 test del aliento. 137 individuos no eran evaluables ya que cumplían alguno de los criterios de exclusión. 481 sujetos $(77,8 \%$ de los test realizados) fueron evaluados. La infección por $H p$ fue detectada en 290 sujetos, lo que supone una prevalencia de 60,3\% (IC95\%: 55,81-64,71).

Entre los individuos evaluados $(\mathrm{n}=481) 175$ eran varones $(36,4 \%)$ y 306 mujeres $(63,6 \%)$. La prevalencia de la infección por $H p$ en función del sexo fue 60,6 y 60,1\% respectivamente, sin detectarse diferencias significativas entre el sexo y la infección ( $\mathrm{p}=0,924)$.

La mediana de edad de la población estudiada fue de 37,5 años (rango 4-82 años). Se establece que existe una asociación estadísticamente significativa entre la infección por $H p$ y la edad ( $\mathrm{z}=-6,098 ; \mathrm{p}<0,001)$. Las diferencias en la edad en función de la infección por $H p$ se muestran en la tabla I. La diferencia de edad entre los pacientes infectados por $H p$ y los no infectados fue de 11 años (diferencia de medianas). Estratificando la variable edad se pudo establecer una asociación lineal estadísticamente significativa entre la edad y la infección por $H p\left(\chi^{2}\right.$ Mantel-Haenszel: $33,31 ; \mathrm{p}<0,001)$ : a mayor edad mayor probabilidad de infección por $H p$.

Tabla I. Mediana y rangos mínimos y máximos en paciente con infección por $\mathrm{Hp}$ y sin ella. Análisis simple

\begin{tabular}{lcccc}
\hline & & \multicolumn{3}{c}{ Edad (años) } \\
\cline { 3 - 5 } Infección & $n$ & Mediana & Mín-Máx & $p$ \\
\hline $\mathrm{Hp}+$ & 290 & 42 & $6-82$ & $<0,001$ \\
$\mathrm{Hp}-$ & 191 & 31 & $4-81$ & \\
\hline
\end{tabular}


La distribución de los sujetos infectados muestra una gran variabilidad en función de la edad de los individuos estudiados. Las prevalencias más elevadas se observan en los tramos de edad comprendidos entre 40-49 y 60-69 años con aproximadamente el $80 \%$ infectados por $H p$ (Fig. 1).

De los 481 individuos evaluados se conoce el nivel de estudios de 169 (35,1\%). De estos el 56,8\% tiene estudios universitarios, el 16,6\% formación profesional y 26,6\% estudios primarios, sin que exista relación en nuestra muestra entre el nivel de estudios y la infección por $\mathrm{Hp}$.

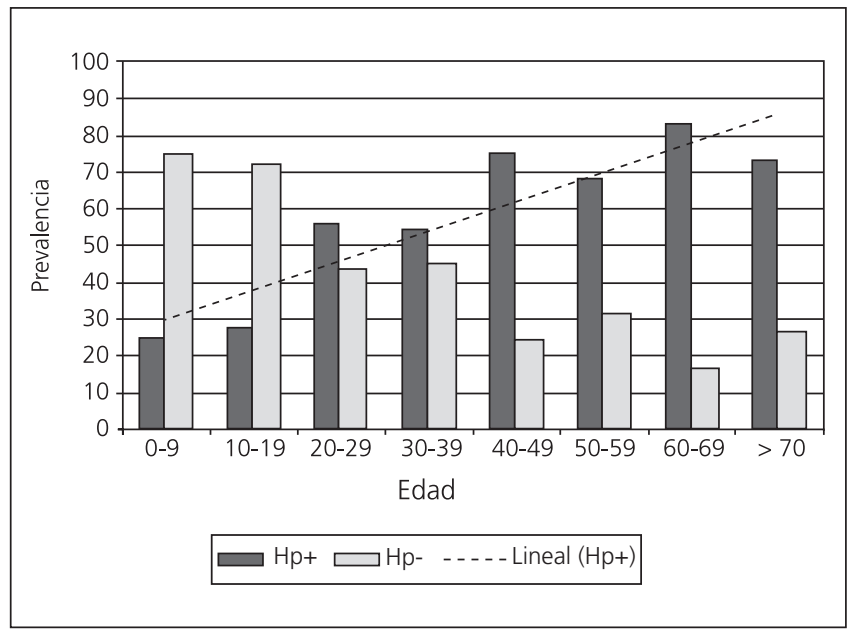

Fig. 1. Distribución de la infección por Hp en función de la edad.

\section{DISCUSIÓN}

La infección por $H p$ es una de las enfermedades infecciosas más frecuentes en el mundo (5) afectando a cualquier edad, sexo, raza o estrato social, aunque con frecuencia variable. Presentamos un estudio epidemiológico sobre la infección por $H p$ en población sana de Madrid. El diagnóstico de la infección por este microorganismo se ha realizado con la prueba del aliento con urea marcada con carbono 13, que se considera por su sensibilidad y especificidad la más fiable para este tipo de estudios $(6,7)$.

La prevalencia de la infección por $H p$ obtenida en nuestro estudio ha sido de $60,3 \%$. Con respecto a otras áreas geográficas nos encontramos lejos de las cifras encontradas en países como Australia (8), Estados Unidos (9), Alemania (10), o Francia (11) donde la prevalencia no supera el $40 \%$, y más cercanos a la prevalencia comunicada en la zona norte de Italia (12) o Argentina (13) con cifras superiores al $60 \%$.

En el continente Africano se observan prevalencias muy elevadas en todas las franjas etarias que superan el $70 \%$ y en algunos países el $80 \%(14,15)$. Por último en India se han comunicado prevalencias (16) muy elevadas similares a las reportadas en los países africanos, mientras que en China y Taiwán la prevalencia se asemeja más a los países más desarrollados $(17,18)$.
Al revisar los estudios realizados en nuestro país las cifras oscilan entre el 84\% obtenido en Guadalajara (19) y las prevalencias más bajas comunicadas en Mallorca y Cataluña con 21 y $38,2 \%$ respectivamente $(20,21)$. Con cifras de prevalencia en torno al $50 \%$ encontramos estudios en País Vasco (22), Asturias (23), Andalucía (24) y Madrid (25). Resultados similares a la prevalencia obtenida en nuestra muestra se obtienen en Canarias (26), Cuenca (27) y Orense (28). La mayoría de estos estudios han utilizado la serología como método diagnóstico de la infección por $H p$ (que frente al test del aliento presenta mayores tasas de falsos positivos). Sólo dos de estos estudios $(28,29)$ junto con el que presentamos, han utilizado el test del aliento como método diagnóstico. También se observan diferencias en la población incluida en los distintos estudios: población general, pacientes hospitalizados o seleccionados en entorno hospitalario o población sin antecedentes de enfermedad digestiva, como es nuestro caso. Creemos que el haber incluido en este estudio sujetos que acuden a una Feria de la Salud no sesga los resultados ya que el interés o la preocupación por la salud no tiene en teoría relación con la tasa de infección.

En nuestra muestra de los 137 individuos no evaluables, 47 tienen antecedentes de cirugía gástrica, tratamiento erradicador previo para $H p$ o consumo de antibióticos/IBP en el mes anterior, circunstancias que hacen poco fiable el test del aliento, y 90 antecedentes de enfermedad ulcerosa o bulboduodenitis sin otros criterios de exclusión.

Si comparamos nuestros datos con el único estudio realizado también en Madrid por Martín de Argila y cols. (25) observamos una prevalencia superior en nuestra muestra comparada con la obtenida diez años antes. Aunque estos dos estudios no son directamente comparables ya que la técnica de diagnóstico de la infección por $H p$ es diferente (seroprevalencia de anticuerpos IgG contra $\mathrm{Hp}$ frente al test del aliento) es reseñable que la prevalencia aumente en este decenio en un entorno socioeconómico desarrollado. Es esperable que a largo plazo la mejora de condiciones socioeconómicas de nuestro medio, acaecidas más tarde en relación con otros países europeos, den lugar a una disminución en la tasa de infección por $\mathrm{Hp}$.

Es característico de la epidemiología de la infección por $\mathrm{Hp}$ una estrecha relación con la edad. Es por ello que las curvas de prevalencia en relación con la edad son las que aportan una mayor información sobre cuándo, y quizás cómo, se adquiere el germen en una población determinada. En los países en vías de desarrollo la mayoría de los niños se infectan durante la infancia y están infectados al llegar a la edad adulta. Por otra parte en los países desarrollados la prevalencia es baja en la infancia y aumenta en relación con la edad. Si comparamos los modelos de infección de los países en desarrollo y los países desarrollados con los datos obtenidos en este estudio, observamos que nos encontramos en una situación intermedia entre ambos patrones, al igual que han descrito otros trabajos en nuestro medio (28) (Fig. 2). 


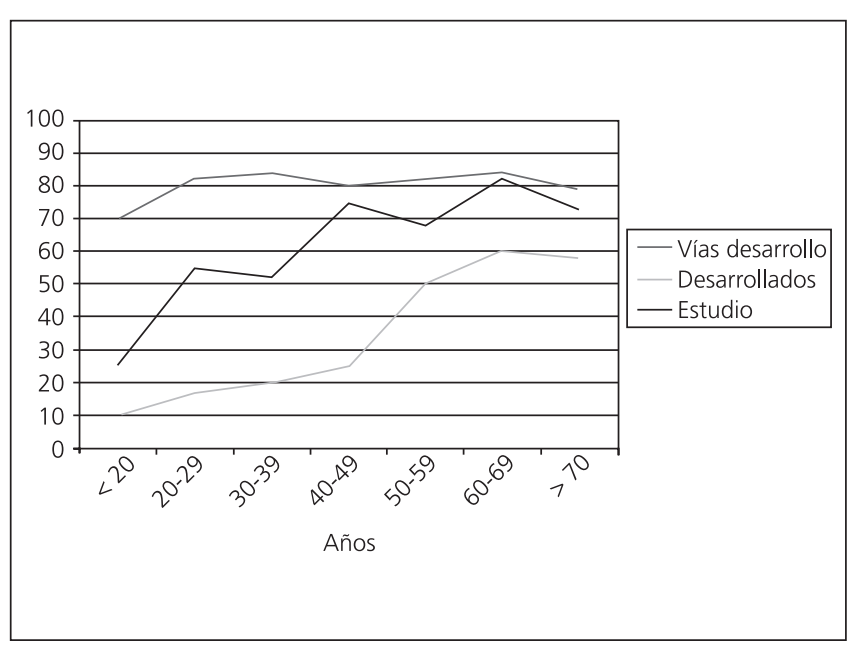

Fig. 2. Extrapolación de las curvas de la prevalencia de la infección por Hp en relación con la edad en países desarrollados en vías de desarrollo (30) y en nuestro estudio.

No hemos encontrado diferencias significativas entre el sexo y la infección, como la mayoría de los estudios. Ni tampoco en nuestro caso en función del nivel de estudios, que conocíamos en el $35,1 \%$ de los sujetos. Con respecto a la edad apreciamos una prevalencia creciente a los largo de la vida alcanzándose las mayores tasas entre los 40-49 y los 60-69 años con prevalencia en torno al 80\%.

En definitiva, nuestro trabajo muestra que la prevalencia de la infección por $H p$, diagnosticada mediante una técnica adecuada para este tipo de estudio, en población sana de Madrid es alta (por encima del 60\%) y va aumentando a lo largo de la vida, siendo máxima entre los 60 y 69 años. No se han observado diferencias globales en función del sexo ni del nivel de estudios. La prevalencia en la Comunidad de Madrid no ha disminuido en la última década y su distribución por edades encuadra nuestra población en una situación intermedia entre los países desarrollados y aquellos en vías de desarrollo.

\section{AGRADECIMIENTOS}

A Isabel Salas, Eladio Sacristán, M. García Alonso, N. López Palacios, J. L. Matas, I. Salueña, L. Domínguez, M. Hernández, J. Delkader, M. Ágreda, R. García, I. Pardo, J. Estellés, Ana I. Romero, M. García Arredondo y Anna Lee Brunner por su colaboración en la realización de encuestas y pruebas.

El estudio se ha realizado en colaboración con la SEPD en el seno de sus actividades en FISALUD.

\section{BIBLIOGRAFÍA}

1. Warrren JR, Marshall B. Unidentified curved bacilli on gastric epithelium in active chronic gastritis. Lancet 1983; I: 1273-5.

2. Go MF. What are the host factors that place an individual at risk hor
Helicobacter pylori asociated disease? Gastroenterology 1997; 113: S5-S20.

3. Covacci A, Telford JC, del Giudice G, Parsonnet J, Rappuoli R. Helicobacter pylori virulence and genetic geography. Science 1999; 284 : 1328-33.

4. Go MF. Review article: natural history and epidemiology of Helicobacter pylori infection. Aliment Pharmacol Ther 2002; 16 (Supl. 1): 3-15.

5. Cave DR, Go M, Cutler A, Goldstein J, Dunn B, Mobley H, et al. Transmission and epidemilogy of Helicobacter pylori. Ann I Med 1996; 100 (5A): 12-8.

6. Savarino V, Vigneri S, Celle G. The ${ }^{13} \mathrm{C}$ breath test in the diagnosis of Helicobacter pylori infection. Gut 1999; 45 (Supl. 1): 118-22.

7. Gisbert JP, Ducons J, Gomollón F, Domínguez Muñoz JE, Borda F, Miño G, et al. Validación de la prueba del aliento con ${ }^{13} \mathrm{C}$-urea para el diagnóstico inicial de la infección por Helicobacter pylori y la confirmación de su erradicación tras el tratamiento. Rev Esp Enferm Dig 2003; 95 (2): 115-20.

8. Peach HG, Pearce DC, Farish SJ. Helicobacter pylori infection in an Australian regional city:prevalence and risk factors. Med J Aust 1997; 167 (6): 310-13.

9. Everhart JE, Kruszon-Moran D, Pérez Pérez GI, Tralka TS, McQuillan G. Seroprevalence and ethnic differences in Helicobacter pylori infection among adults in the United States. J Infec Dis 2000; 181: 1359-63.

10. Breuer T, Sudhop T, Hoch J, Sauerbruch T, Malfertheiner P. Prevalence and risk factors for Helicobacter pylori infection in the western part of Germany. Eur J Gastroenterol Hepatol 1996; 8: 47-52.

11. Megrand F, Brassens-Rabbe MP, Denis F, Belbouri A, Hoa DR. Seroepidemiology of Campylobacter pylori infection in various populations. J Clin Microbiol 1989; 27: 1870-3.

12. Bazzoli F, Palli D, Zagari RM, Festi D, Pozzato P, et al. The LoianoMonghidoro population based study of Helicobacter pylori infection: prevalence by $13 \mathrm{C}$-urea Breath test and associated factors. Aliment Pharmacol Ther 2001; 15 (7): 1001-7.

13. Mattana C, Vega A, Gómez P, Puig de Centorbi O. Serological profile of Helicobacter pylori infection in the population of San Luis (Argentina). Enferm Infecc Microbiol Clin 2004; 22 (4): 227-9.

14. Glupczynski Y, Bourdeaux L, Verhas M, De Pérez C, DeVos D, Devreker $\mathrm{T}$. Use of a urea breath test versus invasive methods to determinate the prevalence of Helicobacter pylori in Zaire. Eur J Clin Microbiol Infect Dis 1992; 11 (4): 322-7.

15. Holcombe C, Omotara BA, Eldrige J, Jones DM. Helicobacter pylori, the most common bacterial infection in Africa: A random serological study. Am J Gastroenterol 1992; 87 (1): 28-30.

16. Graham DY, Adam E, Reddy GT, Agarwal JP, Agarwal R, Evans DJ, et al. Seroepidemiology of Helicobacter pylori infection in India. Comparison of developing and developed countries. Dig Dis Sci 1991; 36 (8): 1084-8.

17. Mitchell HM, Li YY, Hu PJ, Liu Q, Chen M, Du G, et al. Epidemiology of Helicobacter pylori in southern of China: Identification of early chilhood as the critical period of adquisition. J Infect Dis 1992; 166: $149-53$.

18. Lin JT, Wany JT, Wany JH, Wu MS, Lee TK, Chen CJ. Helicobacter pylori inffection in a randomly selected population, healthy volunteers and patients with gastric ulcer and gastric adenocarcinoma. Scand J Gastroenterol 1993; 28: 1067-72.

19. Carballo F, Martínez C, Aldeguer M, García A, Domínguez E, Maltfertheiner P, et al. Infección por Helicobacter pylori en Guadalajara: prevalencia y factores asociados. Rev Esp Enferm Dig 1995; 87 (Supl. 1): 7.

20. Reina J, Salvá F, Alomar P. Análisis de la prevalencia de anticuerpos anti-Campylobacter pylori detectados en la población humana sana. Rev Esp Enferm Dig 1989; 76 (2): 151-4.

21. Navarro M, Calvet X, Font B, Sanfeliu I, Segura F. Prevalence of Helicobacter pylori infection in the Vallés Occidenta, Catalonia. Clin Microbiol Infect 1999; 5 (11): 704-6.

22. Cilla G, Pérez-Talloro E, García Bengoechea M, Marimón JM, Arenas JI. Helicobacter pylori infection: A seroepidemiological study in Guipúzcoa, Basque Country, Spain. Eur J Epidemiol 1997; 13: 945-9.

23. Rodrigo L, Riestra S, Fernández E, Fernández MR, García S, Lauret ME. Estudio epidemiológico de la infección por Helicobacter pylori en la población general de Asturias. Rev Esp Enferm Dig 1997; 89 (7): 511-6. 
24. Caballero AM, Sofos S, Valenzuela M, Martín JL, Casado FJ, Guilarte J. Epidemiología de la dispepsia en una comunidad del sur de España. Prevalencia de la infección por Helicobacter pylori. Rev Esp Enferm Dig 2000; 92 (12): 781-6.

25. Martín de Argila C, Boixeda D, Canton R, Mir N, de Rafael L, Gisbert $\mathrm{J}$, et al. Helicobacter pylori infection in a healthy poulation in Spain. Eur J Gastroenterol Hepatol 1996; 8 (12): 1165-8.

26. Castellot A, Santana E, Orengo JC, Peña L, Santana M, Sierra A, et al. Seroprevalencia de la infección por Helicobacter pylori en las Islas Canarias. Rev Esp Enferm Dig 2001; 23 (Supl. 1): 153.

27. Gónzález JA, Gómez C, García Cano J, Nieto J, Morillas J, Pérez JL, et al. Infección por Helicobacter pylori en población sana en la pro- vincia de Cuenca. Rev Esp Enferm Dig 2003; 95 (Supl. 1): 52.

28. Marcenlle García R, Ganoso Diz P, Sueiro Benavides RA, Fernández Seara J. Prevalencia de la infección por Helicobacter pylori en la población general adulta de la provincia de Ourense. Rev Esp Enferm Dig 2006; 98 (4): 241-8.

29. Rafols A, Solanas P, Ramio G, Suelves N, Rodríguez C, González C, et al. Prevalencia de la infección por Helicobacter pylori en atención primaria de salud. Aten Primaria 2000; 25 (8): 104-11.

30. Martín de Argila C, Boixeda de Miquel D. Epidemiología de la infección por Helicobacter pylori. En: Boixeda de Miquel D, Martín de Argila $\mathrm{C}$, editores. Infección por Helicobacter pylori ¿Más allá del límite? Barcelona; 2001. Tomo 1 p. 47-71. 